\title{
Wavelet analysis of Ising model spin dynamics
}

\author{
George D. J. Phillies \\ Jonathan J. Stott \\ Fairfield University, jstott@fairfield.edu
}

Follow this and additional works at: https://digitalcommons.fairfield.edu/physics-facultypubs Copyright 1995 AIP Publishing

The final publisher PDF has been archived here with permission from the copyright holder.

\section{Peer Reviewed}

\section{Repository Citation}

Phillies, George D. J. and Stott, Jonathan J., "Wavelet analysis of Ising model spin dynamics" (1995).

Physics Faculty Publications. 119.

https://digitalcommons.fairfield.edu/physics-facultypubs/119

\section{Published Citation}

Phillies, George DJ, and Jonathan Stott. "Wavelet analysis of Ising model spin dynamics." Computers in Physics 9 , no. 1 (1995): 97-107.

This item has been accepted for inclusion in DigitalCommons@Fairfield by an authorized administrator of DigitalCommons@Fairfield. It is brought to you by DigitalCommons@Fairfield with permission from the rightsholder(s) and is protected by copyright and/or related rights. You are free to use this item in any way that is permitted by the copyright and related rights legislation that applies to your use. For other uses, you need to obtain permission from the rights-holder(s) directly, unless additional rights are indicated by a Creative Commons license in the record and/or on the work itself. For more information, please contact digitalcommons@fairfield.edu. 


\section{Wavelet analysis of lsing model spin dynamics}

George D. J. Phillies ${ }^{\text {a) }}$ and Jonathan Stott

Department of Physics, Worcester Polytechnic Institute, Worcester, Massachusetts 01609

(Received 11 March 1994; accepted 29 June 1994)

The Burt-Adelson wavelet decomposition was used to analyze the Monte Carlo spin dynamics of a one-dimensional Ising ring. Burt-Adelson wavelets were used because they select for spin domains and domain walls. For wavelet amplitudes $c_{i}^{n}(t)$, we computed mean-square fluctuations $\left\langle\left[c_{i}^{n}(t)\right]^{2}\right\rangle$, spatial correlation functions $\left\langle c_{i}^{n}(t) c_{i+a}^{n}(t)\right\rangle$, and time correlation functions $\left\langle c_{i}^{n}(t) c_{i}^{n}(t+\tau)\right\rangle$. The simulations are in excellent agreement with analytic calculations. The temperature and decomposition order dependencies of the static correlation functions are readily explained by the wavelet support $L$ and the system's correlation length $\xi$. High-order (long-wavelength) wavelet time correlation functions decay exponentially. Low-order wavelet time correlation functions have an approximate two-exponential decay, with a fast temperature-independent relaxation corresponding to the random walk of domain edges and a slow, temperature-dependent relaxation corresponding to domain creation and annihilation. At intermediate temperatures the slow decay rate satisfies the scaling relationship $\Gamma^{-1} \sim\left\langle\left[c^{n}(t)\right]^{2}\right\rangle^{z}$ for $z=1.80$. 01995 American Institute of Physics.

\section{INTRODUCTION}

Wavelets and wavelet transforms may be viewed as generalizations of orthogonal polynomials and Fourier transforms. Over the past decade, a substantial mathematical literature $^{1-9}$ on wavelet techniques, multiresolution analyses, and similar methods has appeared. A more limited literature ${ }^{10-12}$ shows physical applications. Here we demonstrate the utility of wavelet transforms by applying them to a familiar physical problem: the one-dimensional Ising model.

The formal literature on wavelet transforms is extensive. Precursor papers include Burt and Adelson's multiresolution approach to image analysis ${ }^{1 / 2}$ and Daubechies et al.' $\mathrm{s}^{3}$ discussion of nonorthogonal basis sets, notably the Weil-Heisenberg basis and the $a x+b$ (affine) group. ReViews and pedagogical presentations from a mathematical perspective include Strang, ${ }^{4}$ Heil and Walnut, ${ }^{5}$ Daubechies, ${ }^{6}$ and Lawton. ${ }^{7}$ Daubechies ${ }^{6}$ presents an extensive historical análysis of multiresolution analyses and Wavelets; Heil and Walnut ${ }^{5}$ review results of Feichtinger and Groechenig ${ }^{8,9}$ showing the essential unity of Gabor and Wavelet transforms. Actual examples of physical problems analyzed by wavelet methods are few. Arneodo et $a l^{10}$ and Argoul et al. ${ }^{11}$ used wavelet transforms to characterize scaling of electrodeposition clusters. Meneveau ${ }^{12}$ applied wavelet transforms to nonlinear hydrodynamics.

Meneveau ${ }^{12}$ classified wavelet applications as being descriptive or analytic. Descriptive applications use wavelets to filter specific features of data, in the sense that one uses a wave analyzer to filter for power at a given frequency. Analytic applications of wavelets might typically involve a wavelet transformation of the underlying equations of motion and solution for the wavelet components, in the sense that the low Reynolds number Navier-Stokes

T-mail: phillies@wpi.wpi.edu equation can be solved via Fourier transforms and solutions for individual Fourier components.

This article represents a descriptive application of wavelet expansions. Wavelet decompositions of the well-known ${ }^{13}$ static properties of the Ising ring may be calculated analytically. Wavelet-wavelet temporal correlation functions coefficients are not readily accessible analytically for the spin dynamics used here. Wavelet time correlation functions give insight into the Monte Carlo dynamics of an Ising ring.

Section I treats wavelet expansions. Section II describes the Ising model. Section III treats mean-square fluctuations in wavelet amplitudes. Section IV treats spatial correlations of wavelet amplitudes. Section V considers wavelet time-correlation functions. Section VI gives a scaling analysis of wavelet behavior linking static and dynamic correlation functions. The final section presents conclusions. The Appendix shows the apparent lack of utility, for the system analyzed here, in asking that the wavelet basis set be orthonormal.

\section{WAVEHT EXPANSIONS}

Wavelet expansions are a new family of mathematical transforms, hitherto rarely used in physics. Different wavelet transforms have different mathematical properties and emphasize different aspects of the system. A typical wavelet family $F$ is obtained by translation and dilation of a function $f(x)$;

$$
F(a, b ; x)=f(a x-b)
$$

Here $x$, the translation $b$, and the dilation $a$ are real numbers. A wavelet transform of a function $g(x)$ is typically written

$$
\hat{g}(a, b)=\int d x g(x) F(a, b ; x) .
$$


The $\hat{g}(a, b)$ are wavelet components of $g(x)$; they play the role in wavelet methods that Fourier components $\hat{f}(n)$ of a function $f(t)$ play in Fourier theory. Under certain conditions ${ }^{3}$ the wavelet transform is invertible, so that $g(x)$ can be reconstructed from $\hat{g}(a, b)$.

Interesting wavelet properties include:

(1) local support: The support of a function $f(x)$ is the range of values of $x$ for which $f$ is almost everywhere nonzero. The Fourier function $\exp (i n x)$ has global support; it is nonzero almost everywhere. A local change in a function $g(x)$ thus changes almost all of the function's Fourier components $\hat{g}(\omega)$. Wavelet functions $F(a, b ; x)$ are in part interesting if they have local support, so that $F(a, b ; x)$ vanish rapidly (say, as $e^{-x}$ ) outside a limited range. If the wavelet function has local support, a local change in $g(x)$ only modifies a few of the wavelet components $\hat{g}(a, b)$.

(2) local reconstruction: When a wavelet transform with local support can be inverted, alteration of a single wavelet component $\hat{g}(a, b)$ only affects the reconstructed $g(x)$ within a limited range of $x$. In contrast, alteration of a single Fourier component $\hat{g}(\omega)$ in general modifies the reconstruction of $g(x)$ for almost all $x$.

(3) multigridding: The wavelet $F(a, b ; x)$ is a function of real variables $a, b, x$. Sometimes a real $a$ and $b$ are replaced with a discrete $a$ and a $b$ evaluated on a lattice. Multigrid resolutions can be physically attractive because they resolve functions on a discrete series of length scales.

(4) orthogonality: Some families of wavelet representations are orthogonal to their own translates and dilates. If the scalar product is written

$$
\langle F(a, b ; x) F(c, d ; x)\rangle=\int d x f(a x-b) f(c x-d)
$$

one has $\langle F(a, b ; x) F(c, d ; x)\rangle \sim \delta_{a c} \delta_{b d}$, the $\delta$ being the Kronecker delta.

On the other hand, wavelet families may lack:

(1) orthogonality. Some wavelet families are overcomplete, so all members of the family are not orthogonal, even though the wavelet transform is invertible. Members of a wavelet family need not be orthogonal, ${ }^{3}$ but orthogonality is often convenient.

(2) differentiability. Typical wavelets only have a limited number of derivatives.

(3) symmetry. Wavelet functions are typically neither even nor odd.

(4) transparency of source. While a wavelet family is an overcomplete set of functions, individual wavelets are not eigenfunctions of familiar differential operators.

This article uses a single wavelet transform, the BurtAdelson decomposition, 1,2 originally developed for image processing. A rationale for selecting Burt-Adelson wavelets appears in Sec. III. While the decomposition can be written as a single-step projection, computationally $\varnothing\left(N^{1}\right)$, its operations are more transparently described as three processes, namely coarsening with decimation, smoothing, and extraction of "high-frequency" components. In one dimension, the Burt-Adelson decomposition operates on a line of variables $c^{0}(i), i$ indexing the variables, while the superscript 0 labels the $c^{0}$ as the original nondecomposed variables. In the $N$-element Ising ring, the $c^{0}(i)$ are individual spins, with $i \in 0, N-1$.

A coarsening step with decimation is computed

$$
c^{n}(i)=\sum_{k} w(k) c^{n-1}(k+2 i)
$$

Coarsening is iterative, so a $c^{n}$ of arbitrarily high order follows by iterated applications of Eq. (4). The $w(k)$ are constrained so that if the original $c^{0}(i)$ all equal a single constant $c$, the coarsened and smoothed coefficients also all equal $c$, while the high-frequency components $d^{n}(i)$, defined below, vanish. The $w(k)$ are arbitrarily required to be even in $k$. We used coefficients $w(0)=3 / 8, w(1)=w(-1)$ $=1 / 4$, and $w(-2)=w(2)=1 / 16$, shown by Daubechies ${ }^{6}$ to have especially smooth reconstruction properties.

From Eq. (4), the $c^{n}$ are weighted averages over adjacent $c^{n-1}$. Equation (4) also represents a decimation process. $c^{1}(0)$ is obtained from $c^{0}(-2), c^{0}(-1) \ldots, c^{0}(2)$; $c^{1}(1)$ is obtained from $c^{0}(0), c^{0}(1), \ldots, c^{0}(4)$; etc. Each coarsening step doubles the length scale, so the density of the $c^{1}(i)$ is half the density of the $c^{0}(i)$. Correspondingly, the support of the $c^{2}(i)$ in the $c^{0}(i)$ is nearly twice as wide as the support of the $c^{1}(i)$ in the $c^{0}(i)$.

The second step of the decomposition is smoothing; the $c^{n}(l)$ are used to compute the smoothed wavelet components $\bar{c}^{n-1}(i)$. The $\vec{c}^{n-1}(i)$ have twice the density of the $c^{n}(l)$. For $i$ even

$$
\tilde{c}^{n}(i)=2 \sum_{k=-1}^{+1} w(2 k) c^{n+1}\left(\frac{i}{2}+k\right)
$$

and for $i$ odd

$$
\hat{c}^{n}(i)=2 \sum_{k=-1}^{0} w(2 k+1) c^{n+1}\left(\frac{i+1}{2}+k\right)
$$

Factors of 2 maintain normalization. Smoothing is noniterative, each $c^{n}$ being smoothed once to obtain its matching $\bar{c}^{n-1}$. The same $w(n)$ are used for smoothing and coarsening.

The $c^{n}$ and $\tilde{c}^{n}$ are averages, information being discarded in each coarsening or smoothing step. To reconstruct the $c^{0}(i)$ the discarded information is recovered from the high-frequency components $d^{n}(i)$

$$
d^{n}(i)=c^{n}(i)-\tilde{c}^{n}(i)
$$

With increasing $n$, the $\tilde{c}^{n}(i)$ represent smoothings over larger and larger regions, while the $d^{n}(i)$ represent highfrequency components of progressively longer wavelength. High-frequency components are usefully described as projected components by analogy with the projected variables of the Mori-Zwanzig ${ }^{14}$ treatment of statistical mechanics.

In a Burt-Adelson decomposition, a series $c^{0}(i)$ passes Eqs. (4)-(7), generating wavelet amplitudes: the coarsened $c^{n}$, smoothed $\vec{c}^{n}$, and projected $d^{n}$. Wavelet amplitudes are analogous to Fourier amplitudes; they measure the projection of the initial $c^{0}(i)$ on the wavelets. To reconstruct the original $c^{0}$ components, only the $d^{n}$ and the $c^{n}$ of highest $n$ are required. Here we are interested in coarsening and smoothed spin clusters, so we retained the lower-order $c^{n}$ and $\tilde{c}^{n}$ discarded in image processing. 


\section{MONTE CARLO ISING RING DYNAMICS}

We write the Ising model Hamiltonian in the form

$$
H=\sum_{i, j=1}^{N} J_{i j} s_{i} s_{j}+B \sum_{i=1}^{N} s_{i}
$$

where for $N$ spins $s_{i}$ with periodic boundary conditions satisfies $s_{N+j}=s_{j}$. Here (i) the lattice is one-dimensional, (ii) $s_{i}$ is approximated by a single component $s_{i}= \pm 1$, and (iii) $J_{i j}$ is restricted to uniform nearest-neighbor interactions with $J_{12}=J$.

We used model Monte Carlo dynamics under the Metropolis algorithm, ${ }^{15}$ which represents spins interacting with each other and with a heat bath not represented in the Hamiltonian. The bath randomly flips spins, as constrained by detailed balance. Namely, for states $a$ and $b$ having energies $E_{a}$ and $E_{b}$ and statistical weights $W_{a}$ and $W_{b}$ with 'denoting equilibrium values,

$$
\frac{W_{a}^{e}}{W_{b}^{e}}=\exp \left[-\beta\left(E_{a}-E_{b}\right)\right]
$$

Defining first-order interconversion rate constants $k_{a b}$ and $k_{b d}$, at equilibrium the detailed balance condition gives

$$
W_{a}^{e} k_{a b}=W_{b}^{e} k_{b a},
$$

or

$$
\frac{k_{b a}}{k_{a b}}=\exp \left[-\beta\left(E_{a}-E_{b}\right)\right]
$$

Pseudorandom numbers were generated with the Wichman-Hill ${ }^{16}$ algorithm. Procedurally, one spin at a time was selected at random for possible flipping. If flipping the spin increased the system's energy by $\Delta E$, the spin is flipped with probability $P=\exp (-\beta \Delta E)$. If $\Delta E<0$, the spin is always flipped.

Each selection of a spin is a time step, the notional time variable being the number of attempted spin flips with natural units of flips per spin. The Metropolis algorithm is not unique. Other algorithms may satisfy detailed balance but still yield different dynamic behavior. Analytic solution of the one-dimensional Ising model, including dynamic effects under the heat bath algorithm, was given by Glauber. ${ }^{17}$ We used the Metropolis Monte Carlo algorithm, which is not the same as the Glauber's heat bath algorithm. Equilibrium properties are independent of kinetics, so our static but not dynamic properties should agree with Glauber.

Simulations here treat a linear ring of 1024 spins, and time delays $\leqslant 16$ attempted flips per spin. Tests made with smaller or larger systems (e.g., 256, 512, 2048 spins) showed no dependence of our results on system size. The length of a computer run was set at each temperature using Glauber' $\mathrm{s}^{17}$ spin correlation time $\tau_{1}$; simulations were run for $\sim 800 / \tau_{1}$ attempted flips per spin. At our lowest temperatures, $\tau_{1} \approx 10^{-2}$.

\section{MEAN-SQUARE WAVBRT RUCTUATIONS}

A wide variety of possible, wavelet bases are available. To examine the importance of the wavelet basis, Meneveau ${ }^{12}$ analyzed turbulence, applying to the same fluid velocity fields the Haar basis, four- and eight-point Daubechies Wavelets, and a sharp-filtered (in Fourier space) Fourier transform. These wavelets have a common shape, namely short-wavelength oscillations in sign, and are all based on dilations involving twofold changes of length scale. Meneveau found that decompositions based on these wavelets are robust, in that his disparate wavelets yield strikingly similar numerical descriptions of turbulence. The precise choice of wavelet basis was noncritical. The range of circumstances under which one's results are largely independent of basis is not well studied.

A spin system is dominated by spin domains and domain edges. We therefore selected a basis optimized for edge and domain identification. In the Burt-Adelson decomposition, ${ }^{1,2}$ the $d^{n}(i)$ are edge detectors, having large magnitudes near domain edges but vanishing where the $c^{0}(i)$ are constant. The $c^{n}(i)$ and $\tilde{c}^{n}(i)$ are domain detectors; they vanish if spins of +1 and -1 are equally common in their supports.

All wavelet amplitudes follow from the $c^{0}(i)$ and numerical expansion coefficients, e.g.,

$$
c^{n}(i)=\sum_{j} W_{c}(n, i, j) c^{0}(j)
$$

and similarly for the $\tilde{c}^{n}$ and $d^{n}$. The coefficients $W_{c}(n, i, j)$ received an implicit recursive definition in Sec. I. For $n=1$, one has for $i=j$ that $W_{c}(1, i, j)=3 / 8$, for $i=j \pm 1$ one has $W_{c}(1, i, j)=1 / 4$; for $i=j \pm 2$ one has $W_{c}(1, i, j)=1 / 16$; and all other $W_{c}(1, i, j)$ vanish. The $W_{c}(2, i, j)$ are obtained from Eq. (4) by setting $n=2$, using Eq. (12) to replace the $c^{1}$ with the $c^{0}$, and collecting the $c^{0}(j)$. If $B=0$, changing the sign of every spin has no effect on the probability of a state, so $\left\langle c^{n}(i)\right\rangle,\left\langle\tilde{c}^{n}(i)\right\rangle$, and $\left\langle d^{n}(i)\right\rangle$ vanish.

The simplest nonzero wavelet quantities are meansquare wavelet fluctuations $\left\langle\left[c^{n}(i)\right]^{2}\right\rangle,\left\langle\left[\tilde{c}^{n}(i)\right]^{2}\right\rangle$, and $\left\langle\left[d^{n}(i)\right]^{2}\right\rangle$. Equation (12) allows amplitudes to be expressed in terms of spin-spin correlation functions;

$$
\left\langle\left[c^{n}(i)\right]^{2}\right\rangle=\sum_{j, k} W_{c}(n, i, j) W_{c}(n, i, k)\left\langle c^{0}(j) c^{0}(k)\right\rangle
$$

Mean-square wavelet amplitudes may be calculated analytically from Eq. (13), algebraic evaluation of the $W_{c}(n, i, j)$, and the known spin-spin correlation function

$$
\left\langle c^{0}(j) c^{0}(j+n)\right\rangle=\epsilon^{n !},
$$

where $\epsilon=\tanh (\beta J)$, so simulations contribute no new information. Actual analytic calculations are quite tedious, even with computer symbolic manipulation techniques. Analytic results for $n \leqslant 6$ find $\left\langle\left[c^{n}(i)\right]^{2}\right\rangle$ and $\left\langle\left[\bar{c}^{n}(i)\right]^{2}\right\rangle$ are given by polynomials of order $2^{n+2}-4$ and $2^{n+4}-2^{n+2}-4$, respectively. For example,

$$
\begin{aligned}
\left\langle\left[\bar{c}^{0}(i)\right] j^{2}\right\rangle= & \frac{3235}{16384}+\frac{715}{2048} \epsilon+\frac{497}{2048} \epsilon^{2}+\frac{273}{2048} \epsilon^{3} \\
& +\frac{231}{4096} \epsilon^{4}+\frac{35}{2048} \epsilon^{5}+\frac{7}{2048} \epsilon^{6} \\
& +\frac{1}{2048} \epsilon^{7}+\frac{1}{16384} \epsilon^{8}
\end{aligned}
$$

To obtain Eq. (15), Eq. (12) and its analog for $\tilde{c}$ were used to replace $\tilde{c}^{-}(i)$ with a sum of $c^{0}(j)$, leading to terms containing factors $\left\langle c^{0}(j) c^{0}(j+n)\right\rangle$, which are replaced via Eq. (14) with powers of $\epsilon$ which can be resummed. Figures 1 and 2 present $\left\langle\left[c^{n}\right]^{2}\right\rangle$ and $\left\langle\left[d^{n}\right]^{2}\right\rangle$ as functions of inverse 


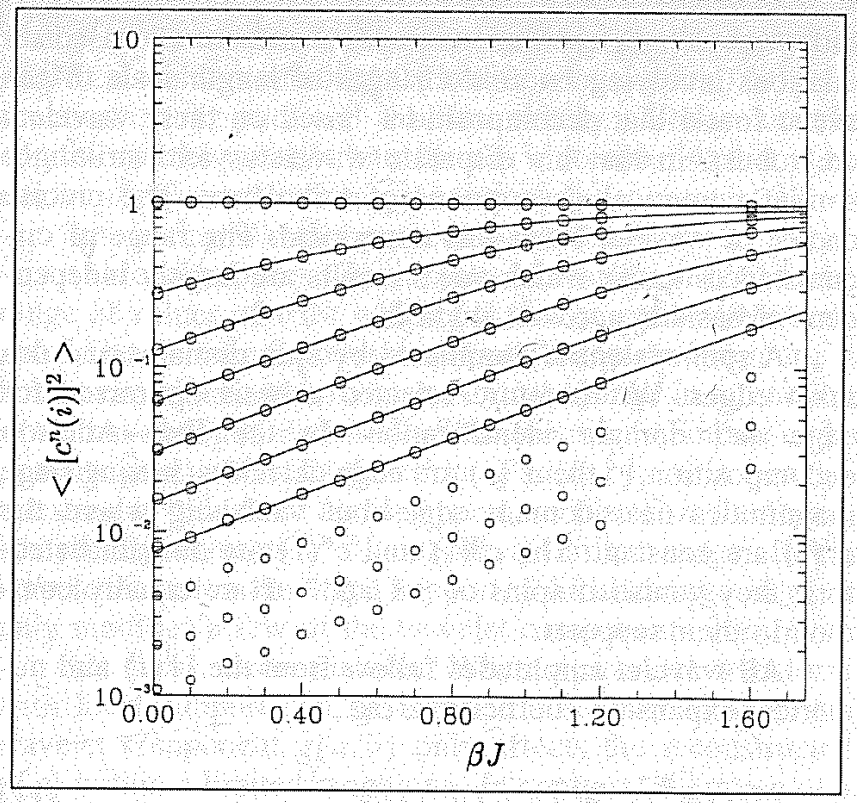

Figure 1. Mean-square wavelet amplitudes $\left\langle\left[c^{n}(i)\right]^{2}\right\rangle$ (log scale) as a function of inverse temperature $\beta J$ for $n=0$ to $n=9$. At fixed $\beta J\left\langle\left[c^{n}(i)\right]^{2}\right\rangle$ decreases monotonically with increasing $n$. Solid lines represent exact analytic calculations; dashed lines guide the eye.

temperature. Points are from Monte Carlo simulations. Solid lines are the analytic calculations of which Eq. (15) is an example. Agreement between simulations and analytic calculations is excellent.

Qualitatively, $\left\langle\left[c^{n}\right]^{2}\right\rangle$ and $\left\langle\left[\tilde{c}^{n}\right]^{2}\right\rangle$ increase as the system becomes colder, approaching unity as $T \rightarrow 0$. This behavior is explained by simple analytic arguments. In the limit $\beta J \rightarrow \infty$ spin domains become extremely large, so all spins in the support of a single wavelet almost certainly have the same value, +1 or -1 ; the $c^{n}$ and $\tilde{c}^{n}$ are therefore almost certainly either +1 or -1 . Mean-square wavelet amplitudes thus go to unity as $T \rightarrow 0$.

High-temperature and large- $n$ wavelet amplitudes are explained by scaling arguments:

First, each wavelet has support $L . L$ is defined such that $c^{n}(0)$ and $\tilde{c}^{n}(0)$ receive contributions from the $c^{0}(i)$ for $-L / 2 \leqslant i \leqslant L / 2$. The support depends on decomposition level $n$ as $L \sim 2^{n}, 1 / L$ is the normalization of wavelet amplitudes, so if all $L+1$ spins in the support of $c^{n}$ have value $+1, c^{n}$ has magnitude $L / L=1$.

Second, spin domains have a temperature-dependent correlation length, so a typical domain contains $\xi$ spins which are correlated with each other, but which are uncorrelated with spins in neighboring domains. In the infinitetemperature limit each spin remains correlated with itself, so $\xi \rightarrow 1$ as $T \rightarrow \infty$.

At infinite temperature, each of the $L$ single-spin domains contributes +1 to the mean-square amplitude. Wavelets have overall normalizations $L^{-1}$, so as $\beta J \rightarrow 0$ the mean-square amplitude is $L \times 1 \times L^{-2} \sim L^{-1} \sim 2^{-n}$, as confirmed quantitatively in Figs. 1 and 2 .

For $\beta J>0 \xi$ and $L$ still determine wavelet amplitudes. If $\xi \ll L$, a wavelet having support $L$ contains $L / \xi$ independent domains. A domain of length $\xi$ contributes $\xi^{2}$ to the mean-square amplitude. The implicit wavelet normalization contributes a factor $L^{-2}$, so in this regime the mean-square amplitude scales as $(L / \xi) \xi^{2} / L^{2} \sim(\xi / L)^{1}$.

$\xi$ is temperature-dependent but $n$-independent, while $L$

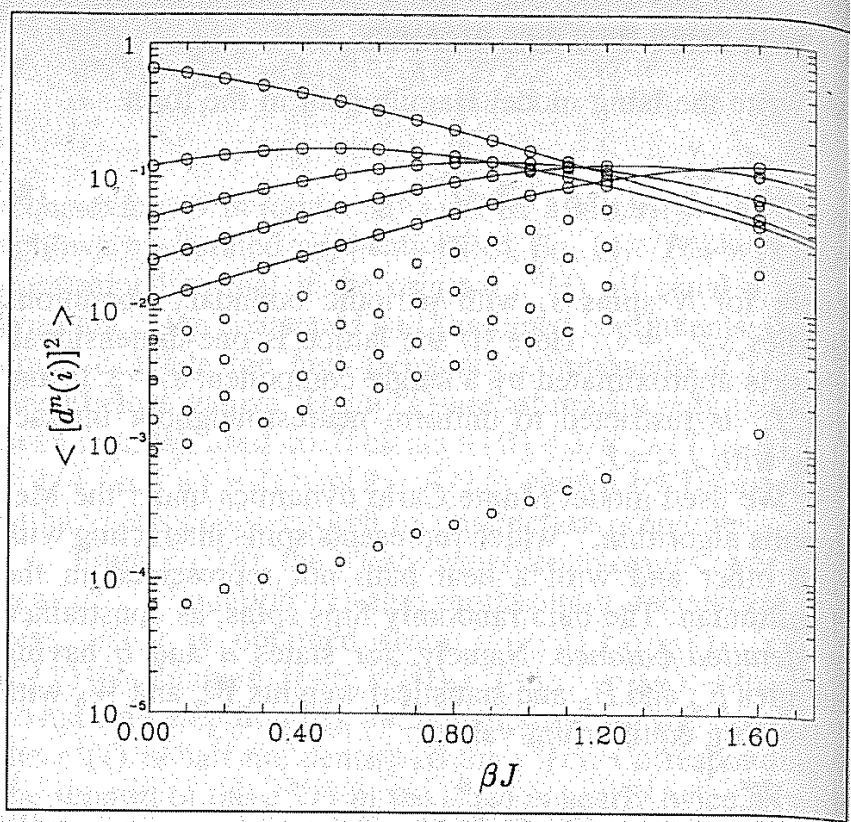

Figure 2. Mean-square wavelet amplitudes $\left\langle\left[d^{n}(i)\right]^{2}\right\rangle(\log$ scale $)$ against $\beta J$ for $n=0$ to $n=8$. At high temperature $(\beta J<0.6)\left\langle\left[d^{n}(i)\right]^{2}\right\rangle$ decreases monotonically with increasing $n$.

depends on $n$ but not $T$. If $\left\langle\left[c^{n}\right]^{2}\right\rangle$ and $\left\langle\left[\tilde{c}^{n}\right]^{2}\right\rangle$ all scale a: $\xi / L$, then (for $\xi \ll L$ ) the ratio of two $\left\langle\left[c^{n}\right]^{2}\right\rangle$ of different $t$ is independent of $T$. This expectation is supported by Figs 1 and 2 ; for large $n$ and small $\beta J$ the $\left\langle\left[c^{n}\right]^{2}\right\rangle$ and $\left\langle\left[c^{n}\right]^{2}\right.$ follow parallel lines.

Figures 1 and 2 and Eq. (14) imply that $\xi$ varies ex ponentially with $\beta$. Table I reports nonlinear least square fits of $\left\langle\left[c^{n}\right]^{2}\right\rangle$ and $\left\langle\left[\tilde{c}^{n}\right]^{2}\right\rangle$ to

$$
\left\langle c^{2}\right\rangle=A \exp (\beta B) \text {. }
$$

From Table I, for $n \geqslant 3$ and $0 \leqslant \beta J \leqslant 1\left\langle\left[c^{n}\right]^{2}\right\rangle$ and $\left\langle\left[\tilde{c}^{n}\right]^{2}\right\rangle$ follow Eq. (16) with $B \approx 1.95$.

From Fig. 2, the $d^{n}$ are somewhat more complicated than the $c^{n}$. We obtained analytic forms for $\left\langle\left[d^{n}(i)\right]^{2}\right\rangle$ for $n \leqslant 4$. At all temperatures there is excellent agreement be tween simulations and analytic forms. The $d^{n}$ are edge detectors, so Fig 2 may qualitatively be understood as a competition of two factors.

First, with increasing $n$, the $d^{n}$ are sensitive to high frequency noise of longer and longer wavelength. To get high-frequency noise of long wavelength, $\xi$ must be large. If $\xi$ is small, high-frequency noise is limited to short wavelengths, so at small $\beta J\left\langle\left[d^{n}(i)\right]^{2}\right\rangle$ falls with increasing $n$. For large $n$ and small $\beta J,\left\langle\left[d^{n}(i)\right]^{2}\right\rangle$ at first increases with increasing $\beta J$, because the increase in $\beta J$ at first increases the extent to which edges are visible at fixed $n$.

Second, as $\beta J$ increases, the domain size increases, so the likelihood of encountering a boundary in the support of a given wavelet falls. As $\beta J \rightarrow \infty$ the support of a $d^{n}$ almost never contains a domain edge. Reducing the number of domain edges per wavelet tends to reduce $d^{n}$, so $\left\langle\left[d^{n}(i)\right]^{2}\right\rangle$ falls when the system becomes cold enough.

\section{WAVEET-WAVEIF SPATIAL CORREATIONS}

The simplest spatial correlations are described by the spinspin correlation function [Eq. (14)], which decays exponen- 
Table I. Nonlinear least square fits of the mean-square wavelet amplitudes $\left\langle\left[c^{n}(i)\right]^{2}\right\rangle$ and $\left\langle\left[c^{n}(i)\right]^{2}\right\rangle$ to $A \exp (\beta B)$.

\begin{tabular}{|c|c|c|c|c|}
\hline$n$ & $A$ & $B$ & $A$ & $B$ \\
\hline 0 & 100 & 0.000 & 0.201 & 1.38 \\
\hline 1 & 0.278 & 1.16 & 0.095 & 1.73 \\
\hline 2 & 0.126 & 1.63 & 0.047 & 1.88 \\
\hline 3 & 0.0617 & 1.84 & 0.0235 & 1.94 \\
\hline 4 & 0.0308 & 192 & 0.0118 & 1.95 \\
\hline 5 & 0.0154 & 195 & $5.87 \times 10^{-3}$ & 1.969 \\
\hline 6 & $7.7 \times 10^{-3}$ & 196 & $2.95 \times 10^{-3}$ & 1.96 \\
\hline 7 & $3.85 \times 10^{-3}$ & 196 & $1.48 \times 10^{-3}$ & 1.95 \\
\hline 8 & $1.93 \times 10^{-3}$ & 1.96 & $1.02 \times 10^{-3}$ & 1.95 \\
\hline 9 & $1.07 \times 10^{-3}$ & 195 & $1.01 \times 10^{-3}$ & 1.95 \\
\hline
\end{tabular}

tially with distance. More elaborate spatial behaviors are potentially revealed by equal-time wavelet-wavelet correlation functions

$$
\begin{aligned}
& C^{n}(a)=\left\langle c^{n}(i) c^{n}(i+a)\right\rangle, \\
& \tilde{C}^{n}(a)=\left\langle\tilde{c}^{n}(i) \bar{c}^{n}(i+a)\right\rangle,
\end{aligned}
$$

and

$$
D^{n}(a)=\left\langle d^{n}(i) d^{n}(i+a)\right\rangle
$$

These correlation functions decrease with increasing a; two physical effects contribute. First, a spin is always correlated with itself, so if the supports of two wavelets overlap, the two wavelets are partially correlated, even as $\beta J \rightarrow 0$. Second, at any finite temperature spin-spin correlations have a nonzero range. Spin contributions to wavelets are partially correlated, even if the supports of the two wavelets are nonoverlapping.

The analysis here is based on Eqs. (4) and (13). Different $w_{n}$ or $W_{c}(n, i, j)$ define other wavelet families. One $\mathrm{can}^{6}$ construct orthonormal wavelets for which $C^{n}(a), \bar{C}^{n}(a)$, and $D^{n}(a)$ vanish at $T=\infty$ and $a>0$, even if the wavelets have overlapping support. As shown in the Appendix, at finite temperature replacing Burt-Adelson wavelets with orthogonal wavelets does not cause $C^{n}(a), \tilde{C}^{\prime \prime}(a)$, or $D^{n}(a)$ to vanish. We used a basis whose form clearly resembles the interesting structures of the Ising ring.

Figures 3 and 4 show representative results for $C^{n}(a)$ and $D^{n}(a)$ at high and intermediate $T$. Data points are simulations; solid lines track analytic calculations. Analytic calculations are only defined for integer $a$; the solid lines are not smooth curves. At all $a$ and $\beta J$ agreement between simulation and algebra is excellent. Note [Fig. 4(a)], the scatter of points with $C^{n}(a) \approx 2 \times 10^{-4}$ and $a \geqslant 5$; these show the simulation noise.

In Figs. 3 and 4, the displacement distance is expressed in decimated spins. For the $n$th order wavelet a displacement of unity represents $2^{n-1}$ times the number of basic spins that is represented by a distance of unity for the $c^{0}(i)$. High- and low-order correlation functions therefore appear to have the same correlation range. For fixed $n \mathrm{C}^{n}(a)$ and $\tilde{C}^{n}(a)$ decrease sharply with increasing $a$. At $\beta J=0.1$, the range over, which the correlation functions are significantly nonzero, is the distance over which wavelet supports overlap. At lower temperatures, long-range spin-spin correlations contribute substantially to the correlation range of $C^{n}(a)$ and $\tilde{C}^{n}(a)$.

Figure 4 presents $D^{n}(a), D^{n}(a)$ has a fixed form at all $n$ and $\beta J$, namely a central peak, a narrow negative region

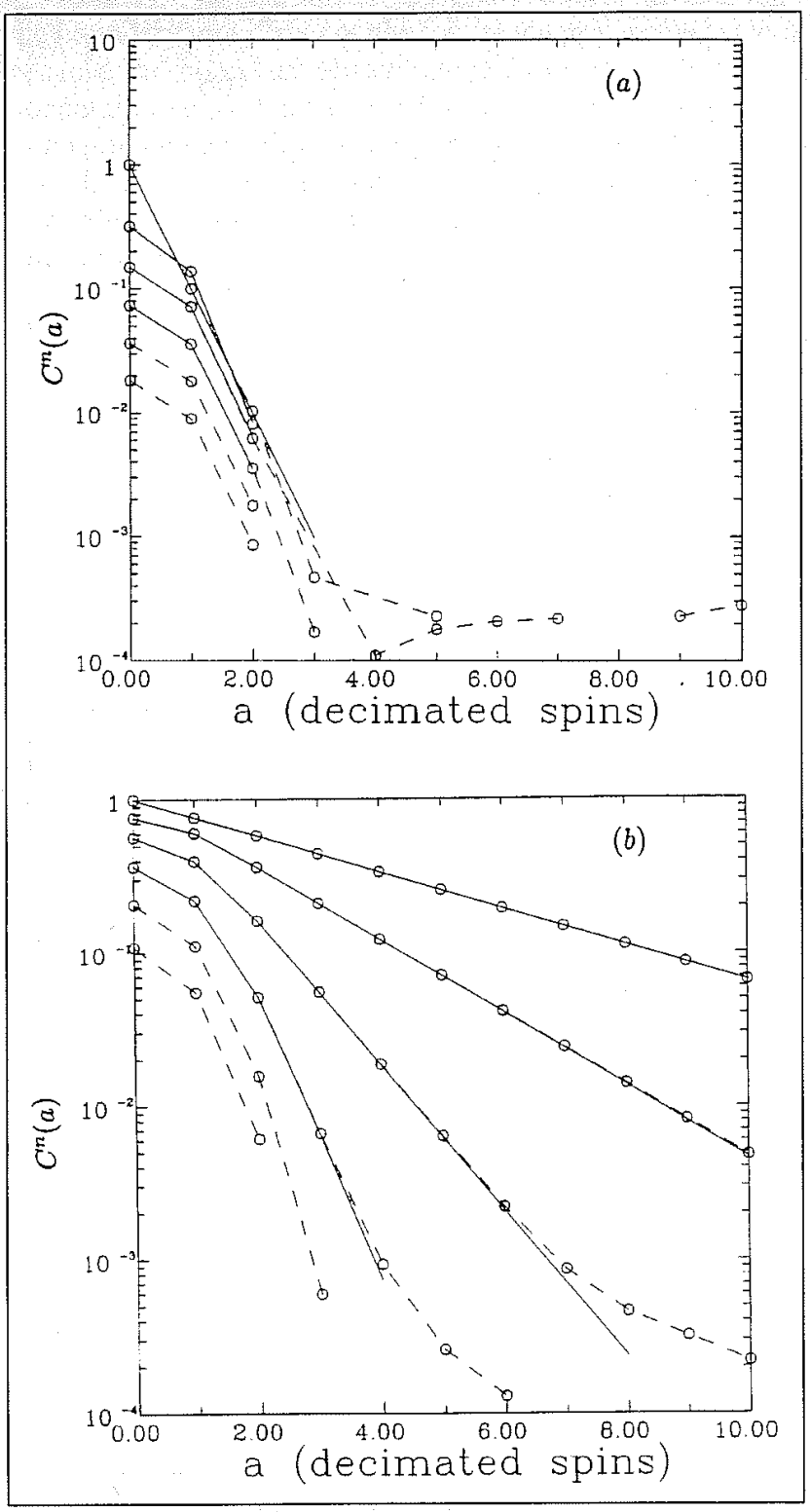

Figure 3. Wavelet-wavelet correlation function $C^{n}(a)$ plotted against separation a $[E q .(17)]$ for $n$ of $0-5$ at temperatures $(a) \beta J=0.1$ and $(b)$ $\beta J=1.0$. Solid lines represent algebraic calculations of $C^{n}(a) . C^{n}(a)$ is defined only for integer a, so lines run point-to-point.

centered at $a=2$, and a weak positive peak near $a=4$ Physically, $D^{n}(a)$ displays the correlation function of an edge with itself; an edge between two spin domains looks nearly the same at all $\beta J$. Up to vertical scale, $D^{n}(a)$ of various $n$ very nearly superpose.

\section{У. WAVEAT-WAVEFT IEMPORAL GORRAUTION FUNCTONS}

Time correlation functions of the total magnetization

$$
M(t)=\sum_{i=1}^{N} c_{i}^{0}(t)
$$

or of an individual spin [i.e., $\langle M(t) M(t+\tau)\rangle$ and $\left\langle c_{i}^{0}(t) c_{i}^{0}(t+\tau)\right\rangle$, where $c_{i}^{n}(t)$ is the value of wavelet $i$ of the $n$th decomposition at time $t]$ give information on spin dynamics. To good approximation, $C_{M}(\tau)$ 


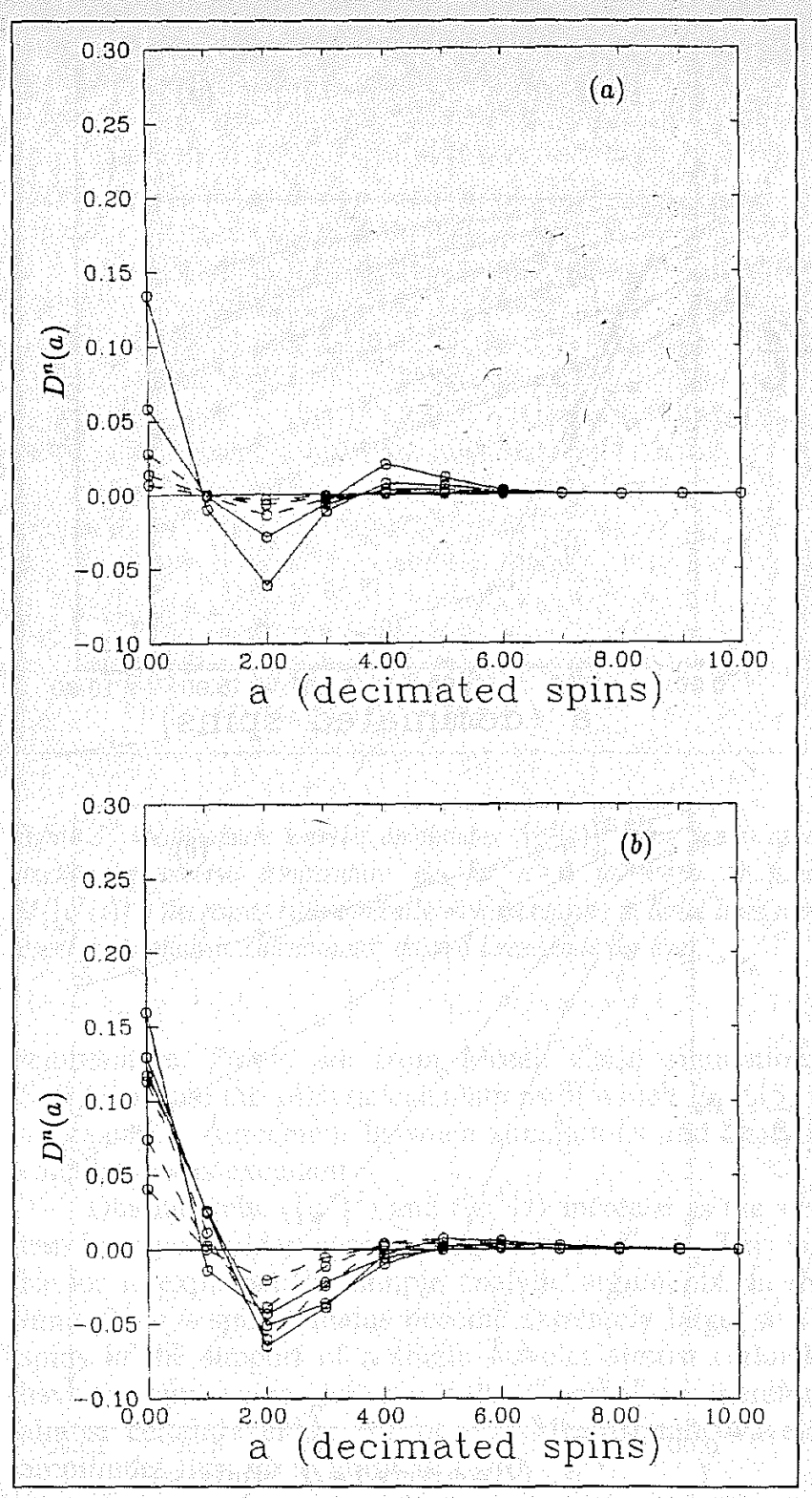

Figure 4. Wavelet-wavelet correlation function $D^{n}(a)$ plotted against a (units of decimated spins), Other details as in Fig. 3.

$=\langle M(t) M(t+\tau)\rangle$ decays exponentially in $\tau$. To test the approximation, we fit our measurements of $C_{M}(\tau)$ at each $\beta J$ to cumulant expansions ${ }^{18}$

$$
C_{M}(\tau)=\sum_{l=0}^{N} \frac{K_{l}^{N}(-\tau)}{l !}
$$

for $N$ of 1 and 2 . The $K_{l}^{N}$ are the cumulants, $K_{0}^{N}$ being the $\tau=0$ intercept, $K_{1}^{N}$ being the decay rate, and the second cumulant $K_{2}^{N}$ being an estimator of the deviation from single-exponential behavior. Cumulant expansions are extensively used in quasielastic light scattering spectroscopy; large-scale Monte Carlo simulations ${ }^{19}$ demonstrate the expansion's reliability under conditions numerically similar to ours. If $C_{M}(\tau)$ is known most accurately at small $\tau$, as is the case here, cumulant expansions are generally superior to moment expansions [e.g, $\left.k_{M l}=\int_{0}^{\infty} d s C_{M}(s) / C_{M}(0)\right]$, because $^{18}$ cumulant expansions implicitly emphasize accurate small- $r$ data, while integral (moment) methods are more sensitive to large- $\tau$ noise.

Table II presents fits to $C_{M}(\tau)$. Increasing $N$ from 1 to
Table II. Time dependence of $\langle M(t) M(t+\tau)\rangle$ as fit to $\exp \left(-K_{1}^{1} \tau\right)$ and to $\exp \left(-K_{1}^{2} \tau+K_{2}^{2} \tau / 2\right.$ ! $)$ at different temperatures.

\begin{tabular}{|c|c|c|c|}
\hline$\beta J$ & $K_{1}^{1}$ & $K_{1}^{2}$ & $100 \frac{\sqrt{K_{2}^{2}}}{K_{1}^{2}}$ \\
\hline 0.01 & 2.00 & 2.07 & 23 \\
\hline 0.05 & 1.67 & 1.72 & 22 \\
\hline 0.075 & 1.65 & 1.62 & 17 \\
\hline 0.1 & 1.45 & 1.49 & 21 \\
\hline 0.2 & 1.06 & 1.06 & 0.3 \\
\hline 0.3 & 0.79 & 0.82 & 24 \\
\hline 0.4 & 0.53 & 0.54 & 14 \\
\hline 0.5 & 0.38 & 0.39 & 12 \\
\hline 0.6 & 0.27 & 0.27 & 15 \\
\hline 0.75 & 0.156 & 0.157 & 11 \\
\hline 0.8 & 0.129 & 0.131 & 21 \\
\hline 0.9 & 0.091 & 0.092 & 17 \\
\hline 1.0 & 0.062 & 0.062 & 18 \\
\hline 1.1 & 0.043 & 0.043 & 20 \\
\hline 1.2 & 0.029 & 0.029 & 8 \\
\hline
\end{tabular}

2 has almost no effect; $K_{2}^{N}$ (here reported as the variance $\left.100 \sqrt{K_{2}^{N} / K_{1}^{N}}\right)$ is only marginally nonzero. $C_{M}(\tau)$ is indeed an exponential. Figure 5 shows decay coefficients (Table II) against $\beta J$. At the resolution of Fig. $5, K_{1}^{1}$ and $K_{1}^{2}$ (jointly denoted $\Gamma$ ) are indistinguishable. The temperature dependence of $\Gamma$ is exponential, namely $\Gamma=2.14$ $\times \exp (-3.53 \beta J)$ with a rms error of $4 \%$. Our simulations used the Metropolis algorithm. In Fig. 5, the solid line represents $K_{1}=2[1-\tanh (2 \beta J)]$ from Glauber' $\mathrm{s}^{17}$ calculation, which implicitly employs the heat bath algorithm. Both algorithms satisfy detailed balance but give different values for $K_{1}^{1}$.

We also determined time correlation functions

$$
\begin{aligned}
& C^{n}(\tau)=\left\langle c_{i}^{n}(t) c_{i}^{n}(t+\tau)\right\rangle, \\
& \bar{C}^{n}(\tau)=\left\langle\tilde{c}_{i}^{n}(t) \tilde{c}_{i}^{n}(t+\tau)\right\rangle,
\end{aligned}
$$

and

$$
D^{n}(\tau)=\left\langle d_{i}^{n}(t) d_{i}^{n}(t+\tau)\right\rangle .
$$

Figures 6 and 7 show $C^{n}(\tau)$ and $D^{n}(\tau) \cdot C^{n}(0)$ and $D^{n}(0)$ are determined by static spin-spin correlations. Time is in natural units: attempted flips per spin. High-temperature plots were terminated at the noise level.

Quantitative interpretation was sought by fitting $C^{n}(\tau)$ and $\bar{C}^{n}(\tau)$ to a single exponential and to sums of two exponentials

$$
C^{\prime \prime}(\tau)=K_{1}\left[K_{3} \exp \left(-K_{2} \tau\right)+\left(1-K_{3}\right) \exp \left(-K_{4} \tau\right)\right] .
$$

It is very difficult to decompose a sum of exponentials unless the decay coefficients differ greatly. Fits were made both with all $K_{i}$ as free parameters and with $K_{2}$ fixed at 2.00 or 1.95 . Two-exponential fits were most reliable at low temperature, where $K_{2} / K_{4}>10$.

At high temperature $C^{n}(\tau)$ decays exponentially. At lower temperatures, $C^{n}(\tau)$ is more complex. For $\beta J>0.3$ high-order decompositions $(n \geqslant 3)$ are consistent with single-exponential behavior, rms errors for two-exponential fits being no better than the one-exponential fits. In the same temperature regime, lower decompositions $(n \leqslant 2)$ are approximately consistent with two-exponential behavior, fits to Eq. (25) being far better than fits to a single expo- 


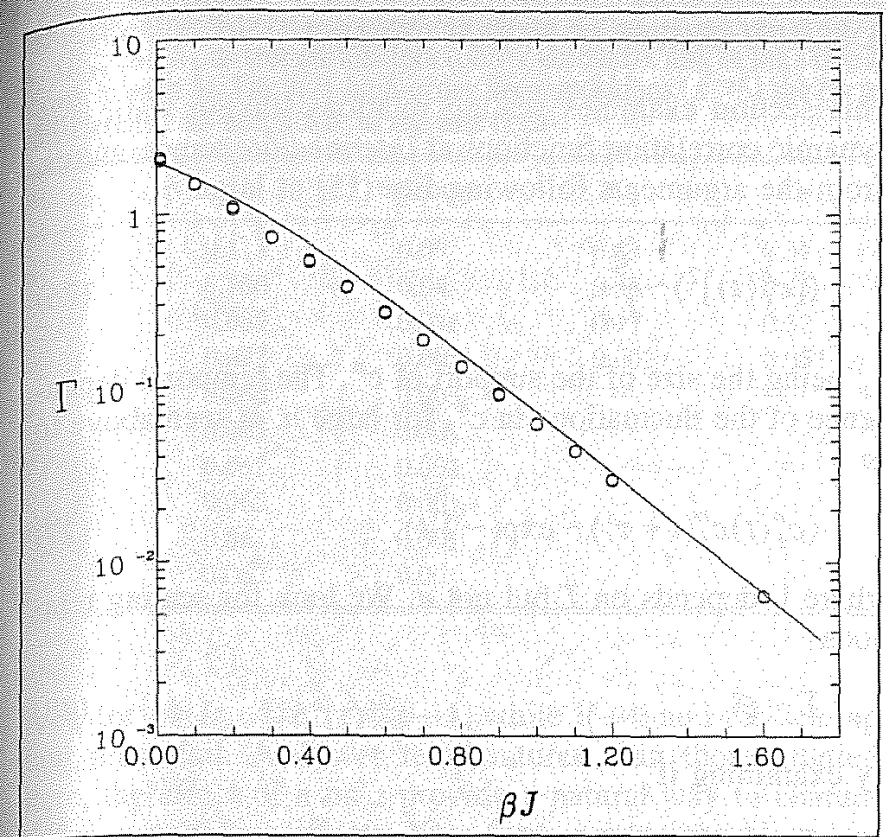

Figure 5. Temperature dependence of the decay rate $K_{1}^{1}=\Gamma$ of the magnetic time correlation function $(M(t) M(t+\tau))=A_{0} \exp \left(-K_{1}^{1} \tau\right)$. Solid line shows predictions of the heat bath algorithm (Ref. 15) to be compared with the Monte Carlo algorithm used here.

nential. Figure 8 shows a representative two-exponential fit; the slow relaxation process is not exactly exponential. Using $K_{2}$ as a free parameter, rather than holding it fixed as $K_{2}=1.95$ or $K_{2}=2.0$, leads to a considerably larger scatter in $K_{4}$ without improvement in the rms error in the fit. With $K_{2}$ held fixed at 1.95 or 2.00 , these values being consistent with $K_{2}$ in the high-temperature limit, $K_{4}$ shows a smooth dependence on $n$, being virtually independent of $n$ for $n \geqslant 4$ and increasing slightly for smaller values of $n$. Table III shows results at $\beta J=1$ and all $n$ for one- and twoexponential fits, $K_{2}$ either being constrained or free.

At all temperatures, and all decompositions at which two exponentials can be discerned, $C^{n}(\tau)$ is consistent with a fast exponential having a temperature-independent $K_{2}$ in the range $1.95-2.00$, together with a slow exponential having a $K_{4}$ that depends strongly on temperature, falling from $K_{4}=1.3$ at $\beta J=0.1$ to $K_{4}=0.062$ at $\beta J=1$. Figure 9 shows $K_{4}$ for $n \geqslant 4$ as a function of $\beta J$.

Measurements of $\bar{C}^{n}(\tau)$ present a picture somewhat simpler than the picture obtained for $C^{n}(\tau)$. At almost all $\beta J$ and $n, \tilde{C}_{i}^{n}(t)$ is very nearly single exponential. Only for $C^{0}(\tau)$ is there weak evidence, a slight increase relative to $n>0$ in the rms error in the single-exponential fits, for a nonexponential decay. For $n=0$, the two-exponential form with $K_{2}=1.95$ gives a better fit than a single exponential. However, the amplitude of the fast exponential is much smaller, typically by a factor of 3 to 5 , for $\tilde{C}^{0}(\tau)$ than for $\mathrm{C}^{0}(\tau)$

It was at first surprising that a dynamics with temperature-dependent rate coefficients has a temperatureindependent relaxation, in addition to the temperaturedependent relaxation seen in $C_{M}(\tau)$. Simple physical considerations explain both relaxations:

First, boundary diffusion yields a temperatureindependent relaxation. The two spins at a boundary between two spin domains necessarily have as neighbors one spin of each sign. Flipping either spin at a boundary moves the boundary, but does not change the energy of the system, because the boundary always contains exactly one pair of nonparallel spins. Under the Metropolis algorithm, the rate of attempted spin-flips is independent of $T$; attempted steps that do not change the system's energy always succeed. The rate at which boundary spins are flipped, which determines the diffusion rate of isolated boundaries, is therefore temperature-independent. Isolated boundary motion changes the number of positive and negative spins within a wavelet, thereby relaxing $C^{n}(\tau)$ and $\bar{C}^{n}(\tau)$ and giving them a temperature-independent decay.

Second, the rate of domain formation, the transformation from one domain to three via a spin-flip in the middle of a domain, falls with decreasing temperature, because flipping a spin within a domain is energetically unfavorable. In the inverse process of domain disappearance, boundaries perform uncorrelated Brownian motion until they collide and annihilate. The rate of domain disappearance, three domains transforming to one via elimination of

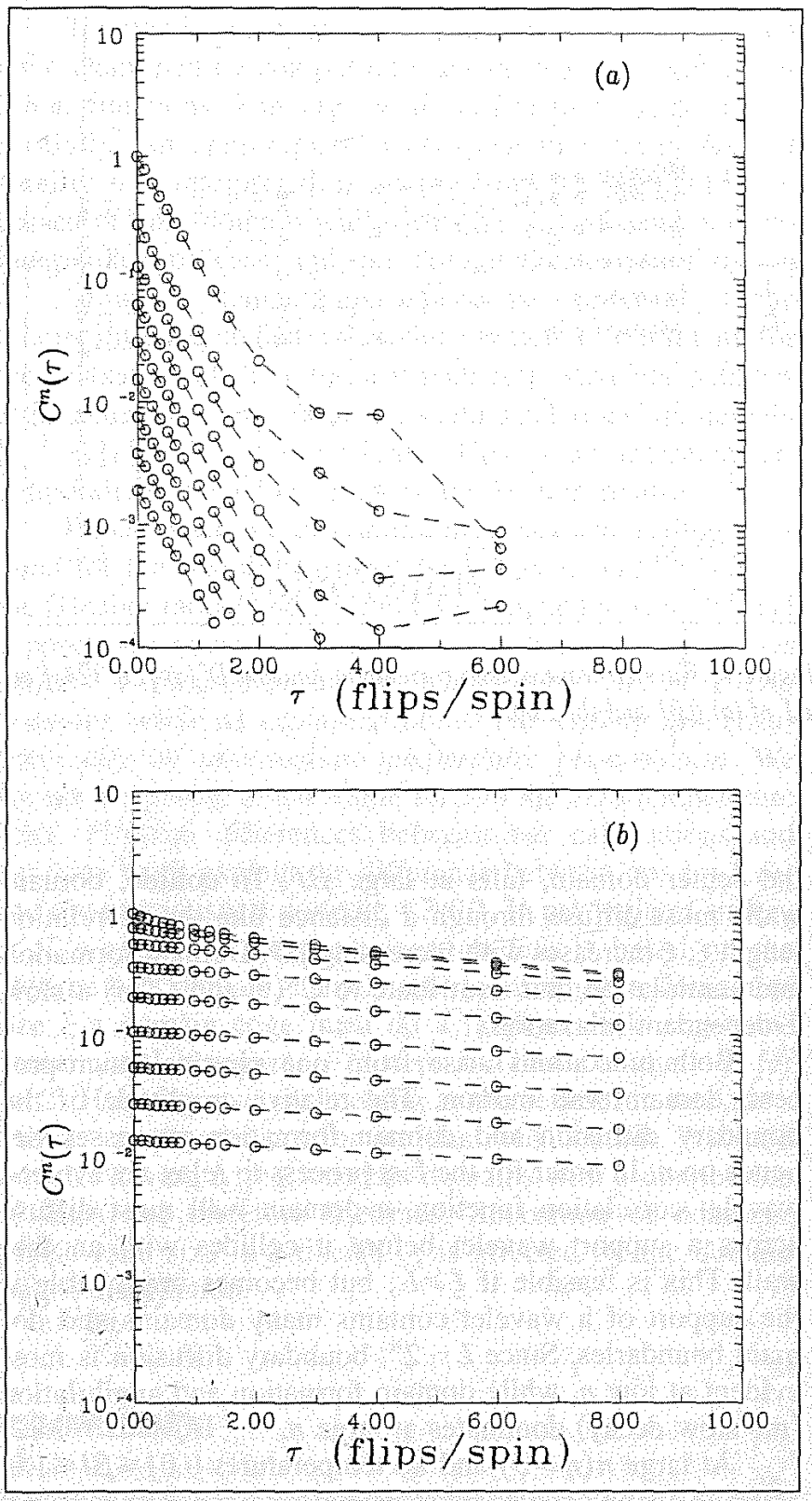

Figure 6. Wavelet-wavelet time correlation function $C^{n}(\tau)[E q .(22)]$ at $\beta J$ of (a) 0.01 and (b) 1.00 . 


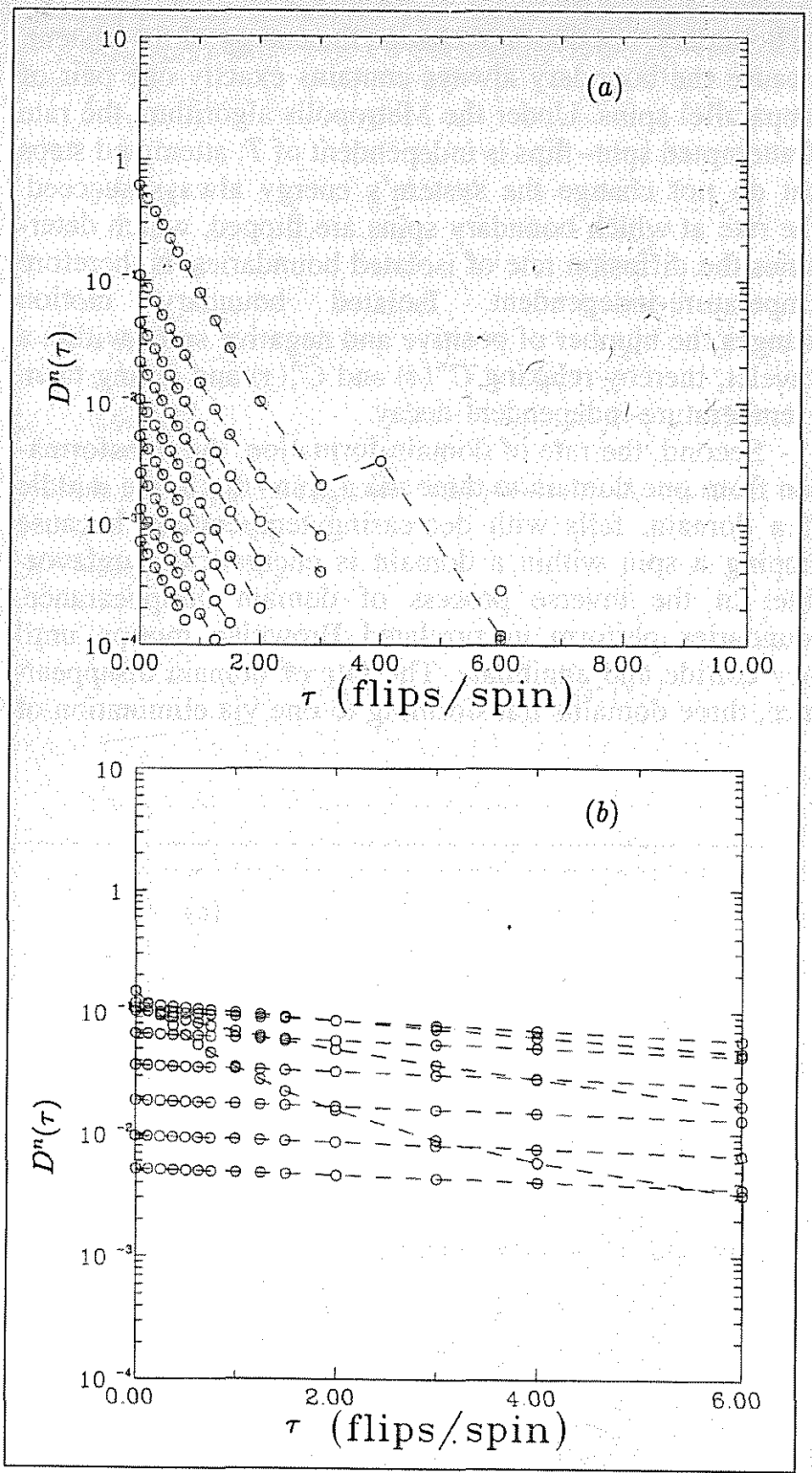

Figure 7. Wavelet-wavelet time correlation function $D^{n}(\tau)[E q$. (24)] at $\beta J$ of (a) 0.01 and (b) 1.00 .

the center domain, falls at large $\beta J$. To collide, domain walls must diffuse through a distance like the correlation length $\xi$; $\xi$ increases with increasing $\beta J$. Domain formation and annihilation thus contribute to $C^{n}(\tau)$ and $\tilde{C}^{n}(\tau)$ a slow $T$-dependent relaxation.

Both relaxations arise from one physical microprocess, domain wall motion. The relative amplitude of the boundary diffusion and domain formation processes depends on $n$. In order for the fast process to relax a waveletwavelet correlation function, a domain wall must diffuse across a support wavelet before it collides with another wall. This is feasible if $\xi \geqslant L$, but becomes improbable if the support of a wavelet contains many domains and domain boundaries. Since $L \sim 2^{n}$, boundary diffusion is most evident at low $n$, while domain formation and annihilation (the slow decay) dominates at large $n$.

At large $n(n \geqslant 3)$ and all temperatures $0.01 \leqslant \beta J \leqslant 1.0$, $D^{n}(\tau)$ is a single exponential whose time constant is within a few percent of the time constants for $M(\tau)$ or $C^{n}(\tau)$. For small $n, D^{n}(\tau)$ is not exponential.

\section{SCALNG BEHAVIOR OF WAVETT DYNAMICS}

This section examines scaling relations linking static and dynamic correlation functions at intermediate temperatures. From the arguments following Eq. (15) at large $n$

$$
\left\langle\left[c_{i}^{n}(t)\right]^{2}\right\rangle \sim \frac{\xi}{L_{n}},
$$

$L_{n}$ being the size of the support of $c^{n}$. The temporal dependence of the fluctuations in $c^{n}$, for large $n$, is seen above to be

$$
\left\langle c_{i}^{n}(t) c_{i}^{n}(t+\tau)\right\rangle \sim \exp (-\Gamma t)
$$

where $\Gamma$ depends on $T$ but not $n$. We look for scaling relations

$$
\Gamma \sim \xi^{z}
$$

by examining if

$$
\Gamma \sim\left\langle\left[c_{i}^{n}(t)\right]^{2}\right\rangle^{z}
$$

Figure 10 is a $\log -\log$ plot of $\Gamma^{-1}$ against $\left\langle\left[c_{i}^{n}(t)\right]^{2}\right\rangle$. Solid lines correspond to fits for $z$. While there is some deviation from a power-law dependence at high temperature (near the bottom of Fig. 10), down to the lowest temperatures examined scaling-law behavior is evident. For the longest wavelengths (largest $n$ ), one obtains $z=1.80$.

Note that the scaling law of Eq. (29) is intrinsicially an intermediate-temperature phenomenon. As $T \rightarrow 0$, one expects $\Gamma^{-1} \rightarrow 0$, but in the same low temperature limit $\left\langle\left[c_{i}^{n}(t)\right]^{2}\right\rangle \rightarrow 1$. Thus, in the limit $\beta J \rightarrow \infty$ Eq. (29) cannot be satisfied. This failure of the scaling law near $T \rightarrow 0$ corresponds to a physical effect expected for real material systems, namely that scaling laws involving $\xi$ must fail when $\xi$ exceeds the size of the system. For a wavelet expansion, the relevant length is not the size of the system but the support $L_{n}$ of the wavelets. For $\xi \ll L_{n}$, the scaling law can be cor-

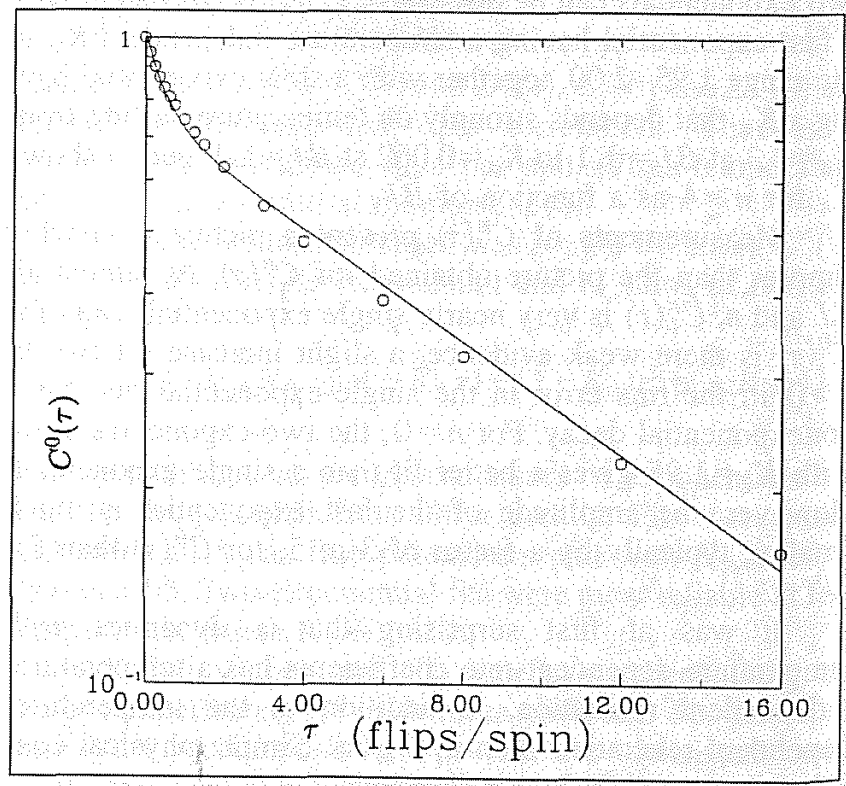

Figure 8. Relaxation of $\log \left[C^{0}(\tau)\right]$ at $\beta J=1.0$, showing approximate $(3 \%$ fractional rms error) two-exponential relaxation. The fit line is $C^{0}(\tau)=1.03[0.264 \exp (-1.95 \tau)+0.736 \exp (-0.101 \tau)]$. 
Table III. Time dependence of $\left\langle c^{n}(t) c^{n}(t+\tau)\right\rangle$ at $\beta J=1$, as fit to $\exp (-\Gamma \tau)$ (right-hand columns), to $K_{1}\left[K_{3} \exp \left(-K_{2} \tau\right)+\left(1-K_{3}\right) \exp \left(-K_{4} \tau\right)\right]$ with $K_{2}, K_{3}$, and $K_{4}$ as free parameters (center columns), and to the same function with $K_{3}$ and $K_{4}$ as free parameters and $K_{2}=1.95$ fixed, including rms errors in each fit. For very high-order decompositions $(n \geqslant 5)$ the correlation function is so close to a single exponential that fits made with $K_{2}$ fixed find virtually no amplitude in the fast decay.

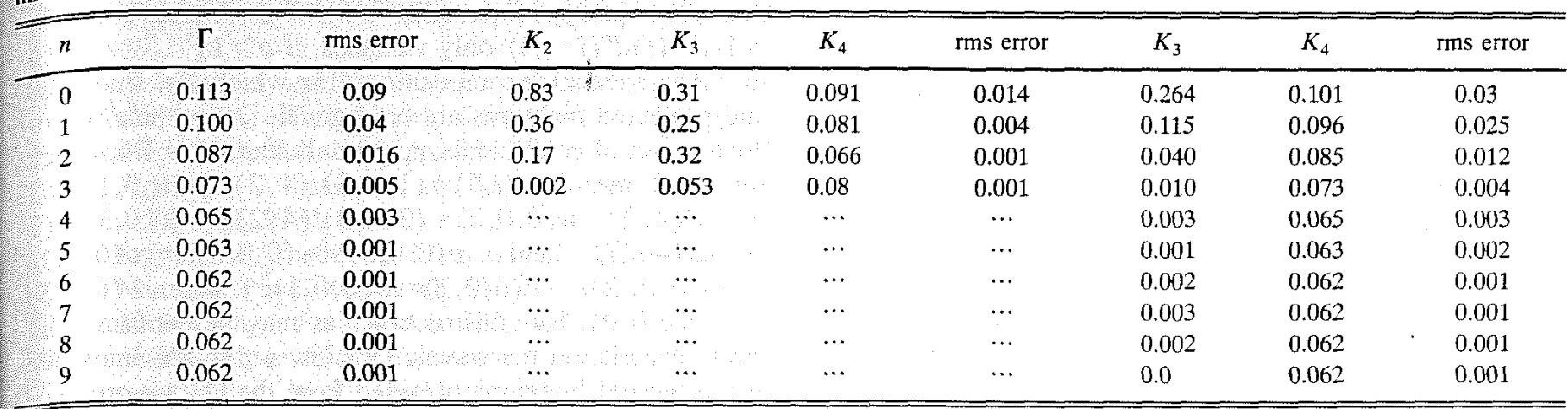

rect; for $\xi \gg L_{n},\left\langle\left[c_{i}^{n}(t)\right]^{2}\right\rangle \rightarrow 1$, while $\Gamma \rightarrow 0$ and the scaling law must fail. At lower temperatures than those studied here, the effect of $n$ on $z$ provide a natural way to characterize system size effects. Note further that the scaling law is only unambiguous because we are examining wavelet amplitudes of high order $n$. At smaller $n C^{n}(\tau)$ is not a simple exponential; at larger $n$ the scaling law becomes clear.

\section{SUMMARY}

The Burt-Adelson ${ }^{1,2}$ decomposition was applied to study spin dynamics of a one-dimensional Ising ring. The BurtAdelson decomposition exemplifies the wavelet expansions $^{6}$ that have recently become of substantial ${ }^{3-12}$ mathematical and scientific interest. Relative to other expansions, the Burt-Adelson procedure uses basis functions that should be sensitive to domains and edges, spin domains and domain edges being physical features that are reasonably expected to be important in models for magnetic systems.

Mean-square amplitudes and spatial correlation functions were determined for coarsened, smoothed, and projected wavelet amplitudes. Static correlation functions were

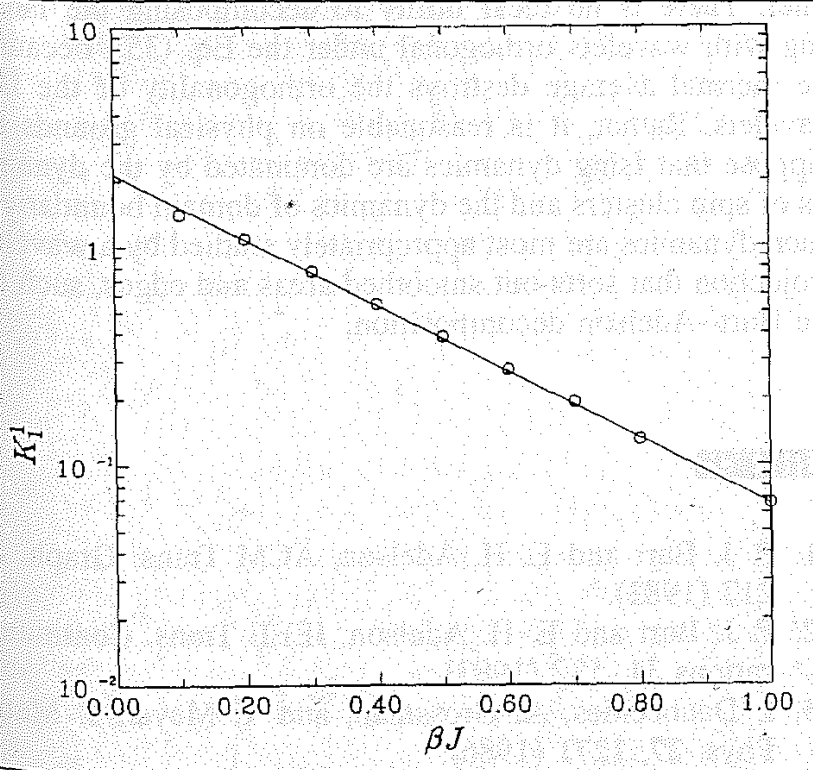

igure 9. Temperature dependence of the relaxation rate $K_{1}^{1}$ of the $C^{\prime \prime}(\tau)$ or $n \geq 4$, showing the near-exponential dependence of $K_{1}^{1}$ on $\beta J$. also computed analytically. Excellent agreement is seen between simulations and analytic calculations. Simple physical considerations explain how the correlation functions are determined by $T, n$, and $a$ via the wavelet support $L$ and a correlation length $\xi$.

Temporal dynamics of the one-dimensional Ising ring were examined by computing wavelet-wavelet time correlation functions. For large $n$, these functions decay exponentially. An approximate two-exponential decay seen at smaller $n$ is interpreted as arising from the separable processes of spin-domain wall diffusion (a fast, temperatureindependent process) and spin-domain creation/annihilation (a slower, temperature-dependent process). The temperature-dependent relaxation, which is dominant for the relaxation of the longest-wavelength wavelets, satisfies at intermediate temperatures a scaling relationship, namely $\Gamma_{n}^{-1} \sim\left\langle\left[c_{i}^{n}(t)\right]^{2}\right\rangle^{z}$ for $z=1.80$. This is an intermediatetemperature, not a low-temperature, scaling relation.

Previous studies of correlation times and scaling relations for the one-dimensional Ising model find ${ }^{20} z=2$ for the Glauber model and $2 \leqslant z \leqslant 4$ for kinetic models ${ }^{21-23}$ that suppress domain wall motion. We emphasize that previous efforts $^{20-23}$ focused on the low-temperature limit of scaling behavior, while as explained above our scaling law is intrinsically an intermediate-temperature phenomenon. We are not proposing a new value for $z$ in the zero-temperature limit. Physical differences between our calculations and previous work explain the difference between our $z=1.80$ and the previously reported $z=2.00$. In particular, Cordery et al.'s argument for the relaxation rate is based on the lifetime of a domain, namely that to eliminate a domain of size $\xi$ a domain edge must do a random walk across the domain, which requires $\sim \xi^{2}$ steps and hence a time $\sim \xi^{z}$ for $z=2$. However, we are looking at the relaxation rate for a wavelet amplitude, not for an identified domain. Within a wavelet having $L_{n} \gg \xi$, one has domains with a distribution of sizes and therefore lifetimes. Relaxation of domains, whose total size is a fixed $L_{n}$, is affected by the domain length distribution.

\section{ACKNOWLEDMENT}

The partial support of this work by the National Science Foundation under Grant No. CHE91-15637 is gratefully acknowledged. 


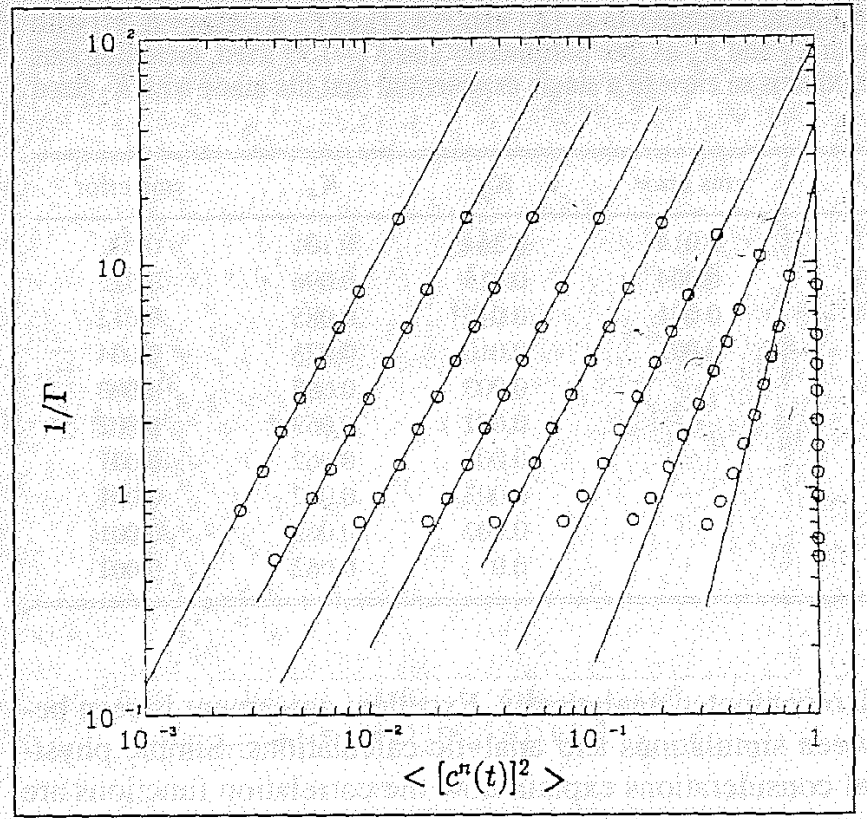

Figure 10. Relaxation time $\Gamma^{-1}$ of wavelets $c^{n}(t)$ of order $n$, plotted as a function of the mean-square fluctuations $\left\langle\left[c^{n}(t)\right]^{2}\right\rangle$. The order of the wavelet decomposition increases from right to left, the $n=0$ points lying on the right-hand axis, while the most left-hand points are for $n=8, \beta J$ increases towards the upper right-hand corner. Solid lines are scaling-law fits (see text).

\section{APPENDY, COMPARISON OF BUBT-ADESON AND ORTHOGONUL WAVAET BASES}

A nominally potent criticism of our choice of the BurtAdelson decomposition ${ }^{1,2}$ is that the resulting wavelets are not orthogonal to each other or to their translates. Here we show that this criticism is inappropriate. For the linear Ising lattice, an arbitrary set of smoothed and projected wavelets can be written in terms of the original spins $e_{i}$ as

$$
\begin{aligned}
& \bar{c}^{n}(i)=\sum_{j} w(n, i, j) e_{j} \\
& d^{n}(i)=\sum_{k} v(n, i, k) e_{k} .
\end{aligned}
$$

Equations (30) and (31) may be viewed as descriptions of the $\tilde{c}^{n}$ and $d^{n}$ as vectors in a linear vector space $V$, whose basis vectors are the $e_{j}$ and $e_{k}$. The $w(n, i, j)$ and $v(n, i, k)$ are expansion coefficients, which for the Burt-Adelson decomposition are defined implicitly by Eqs. (4)-(7).

Smoothed and projected wavelets would be said to be orthogonal if they satisfied orthogonality conditions on their translates

$$
\begin{aligned}
& \left\langle\tilde{c}^{n}(i) \tilde{c}^{n}(i+a)\right\rangle=C \delta_{a 0}, \\
& \left\langle d^{n}(i) \tilde{c}^{n}(i+a)\right\rangle=0, \quad \forall a,
\end{aligned}
$$

and

$$
\left\langle d^{n}(i) d^{n}(i+a)\right\rangle=C^{\prime} \delta_{a 0},
$$

where $C$ and $C^{\prime}$ are constants fixed by the definition of the wavelets, $a$ is an integer, and $\delta_{a 0}$ is the Kronecker delta. The composition $\langle\cdots\rangle$ maps $\mathbf{V} \otimes \mathbf{V} \rightarrow R, R$ being the real numbers, the composition process being defined on the basis vectors as

$$
\left\langle e_{i} e_{k}\right\rangle=\delta_{i k}
$$

By direct computation, the Burt-Adelson decomposition does not satisfy Eqs. (32)-(34). For example, for $n$ $=1\left\langle d^{n}(i) d^{n}(i+a)\right\rangle$ only vanishes, if $a \geqslant 11$.

There exist decompositions for which the smoothed and projected functions are orthogonal. Daubechies ${ }^{6}$ intro. duced a set of coefficients, commonly known as D4, which for $n=1$ are $w(0,0,0)=(1+\sqrt{3}) /(4 \sqrt{2}), w(0,0,1)=(3$ $+\sqrt{3}) /(4 \sqrt{2}), w(0,0,2)=(3-\sqrt{ } 3) /(4 \sqrt{ } 2), w(0,0,3)=(1$ $-\sqrt{3}) /(4 \sqrt{ } 2), \quad$ and $\quad v(0,0,0)=w(0,0,3), \quad v(0,0,1)$ $=-w(0,0,2), \quad v(0,0,2)=w(0,0,1), \quad$ and $v(0,0,3)$ $=-w(0,0,0)$. By construction, as may be confirmed by direct calculation for wavelets of low order, the smoothed and projected functions obtained from the D4 are orthogo. nal under the scalar product of Eq. (35).

For applications in statistical mechanics, $\langle\cdots\rangle$ is appropriately interpreted as a thermal average with GibbsBoltzmann weighting. In the linear Ising ring, the $c^{0}(i)$ are individual spins, so the left-hand side of Eq. (35) is appropriately interpreted as an equal-time spin-spin correlation function, shown analytically by Glauber ${ }^{15}$ to be

$$
\left\langle e_{i} e_{j}\right\rangle=\eta^{|i-j|} \text {. }
$$

where $\eta=\tanh (\beta J)$.

If Eq. (36) replaces Eq. (35), the smoothed and projected functions obtained either from D4 or from the BurtAdelson decomposition are not orthogonal to each other or to their translates, at any finite $T$. Cross-correlation functions for the Burt-Adelson decomposition and $(\cdots)$ the thermal average were computed in Secs. III and IV. By direct calculation from the $\mathrm{D} 4$ coefficients and composition of the $e_{i}$ via Eq. (36) one finds, e.g.,

$$
\begin{aligned}
& \left\langle c^{1}(0) c^{1}(1)\right\rangle=\eta+2 \eta^{2}+1.125 \eta^{3}-\eta^{5} / 8 \\
& \left\langle d^{1}(0) d^{1}(1)\right\rangle=-\eta+2 \eta^{2}-1.125 \eta^{3}-\eta^{5} / 8 \\
& \left\langle c^{1}(0) d^{1}(0)\right\rangle=\sqrt{3} / 4\left(\eta-\eta^{3}\right)
\end{aligned}
$$

Under a thermal average, the $c^{n}$ s and $d^{n}$ s generated from D4 are not orthogonal to their own translates or to each other. There is no clear utility to decomposing the Ising ring with wavelets orthogonal under the Eq. (35), because the thermal average destroys the orthogonality of the D4 wavelets. Rather, it is reasonable on physical grounds to suppose that Ising dynamics are dominated by the dynamics of spin clusters and the dynamics of domain boundaries. Such dynamics are most appropriately studied by a wavelet projection that sorts out smoothed areas and edges, such as the Burt-Adelson decomposition.

\section{REFEBENGES}

1. P. J. Burt and E. H. Adelson, ACM Trans. Graph. 2, 217 (1983).

2. P. J. Burt and E. H. Adelson, IEEE Trans. Communications 31, 532 (1983).

3. I. Daubechies, A. Grossman, and Y. Meyer, J. Math. Phys. 27, 1271 (1986).

4. G. Strang, SIAM Rev. 31, 614 (1989).

5. C. E. Heil and D. F. Walnut, SIAM Rev. 31, 628 (1989) 
6. I. Daubechies, Commun. Pure Appl. Math. 41, 909 (1988).

7. W. M. Lawton, J. Math. Phys. 31, 1898 (1990).

8. H. Feichtinger, Rocky Mountain J. Math. 19, 113 (1989). 3

9. H. Feichtinger and K. Groechenig, Lecture Notes in Mathematics, edited by M. Cwikel et al. (Springer, Berlin, 1988), Vol. 1302, pp. 52-73.

10. A. Arneodo, G. Grasseau, and M. Holschneider, Phys. Rev. Lett. 61, 2281 (1988).

11. F. Argoul, A. Arneodo, J. Elezgaray, G. Grasseau, and R. Murenzi, Phys. Rev. A 41, 5537 (1990).

12. C. Meneveau, J. Fluid Mech. 232, 469 (1991).

13. K. Kawasaki, in Phase Transitions and Critical Phenomena, edited by C. Domb and M. S. Green (Academic, New York, 1972), Chap. 11.

14. H. Mori, Progr. Theor. Phys. (Kyoto) 33, 423 (1965);
34, 399 (1965); R. Zwanzig, Ann. Rev. Phys. Chem. 16, 67 (1965).

15. N. Metropolis, A. W. Rosenbluth, M. N. Rosenbluth, A. H. Teller, and E. Teller, J. Chem. Phys. 21, 1087 (1953).

16. B. Wichmann and D. Hill, Byte 12(5), 127 (1987).

17. R. J. Glauber, J. Math. Phys. 4, 294 (1962).

18. D. E. Koppel, J. Chem. Phys. 57, 4814 (1972).

19. G. D. J. Phillies, J. Chem. Phys. 89, 91 (1988).

20. R. Cordery, S. Sarker, and J. Tobochnik, Phys. Rev. B 24, 5402 (1982).

21. U. Deker and F. Haake, Z. Phys. B 35, 281 (1979).

22. J. C. Kimball, J. Stat. Phys. 21, 289 (1979).

23. F. Haake and K. Thol, Z. Phys. B 40, 219 (1980).

24. J. Tobochnik, S. Sarker, and R. Cordery, Phys. Rev. Lett. 46, 1417 (1981). 